\title{
Clustering a building stock towards representative buildings in the context of air-conditioning electricity demand flexibility
}

\author{
D. Patteeuw ${ }^{\mathrm{a}, \mathrm{c}}$, G.P. Henze ${ }^{\mathrm{b}}$, A. Arteconi ${ }^{\mathrm{a}, \mathrm{d}}$, C.D. Corbin ${ }^{\mathrm{b}}$, L. Helsen ${ }^{\mathrm{a}, \mathrm{c} *}$ \\ ${ }^{a}$ University of Leuven, Department of Mechanical Engineering, Celestijnenlaan 300 - bus 2421, 3000 \\ Leuven, Belgium; ${ }^{b}$ Department of Civil, Environmental and Architectural Engineering, University of \\ Colorado, Boulder, CO 80309 USA; ${ }^{c}$ EnergyVille, Thor Park 8310, 3600 Genk, Belgium; ${ }^{d}$ Universitá degli \\ Studi eCampus, via Isimbardi 10, Novedrate (CO) 22060, Italy;
}

(Received 00 Month 20XX; final version received 00 Month 20XX)

Keywords: Building stock diversity; Air-conditioning; Aggregation; Clustering; Demand flexibility;

\begin{abstract}
Energy modeling for the prediction of energy use in buildings, especially under novel energy management strategies, is of great importance. In buildings there are several flexible electrical loads which can be shifted in time such as thermostatically controllable loads. The main novelty of this paper is to apply an aggregation method to effectively characterize the electrical energy demand of air-conditioning (AC) systems in residential buildings under flexible operation during demand response and demand shaping programs. The method is based on clustering techniques to aggregate a large and diverse building stock of residential buildings to a smaller, representative ensemble of buildings. The methodology is tested against a detailed simulation model of building stocks in Houston, New York and Los Angeles. Results show good agreement between the energy demand predicted by the aggregated model and by the full model during normal operation (normalized mean absolute error, NMAE, below 10\%), even with a small number of clusters (sample size of 1\%). During flexible operation, the normalized mean absolute error rises (around $20 \%$ ) and a higher number of representative buildings becomes necessary (sample size at least 10\%). Multiple cases for the input data series were considered, namely by varying the time resolution of the input data and the type of input data. These characteristics of the input time series data are shown to play a crucial role in the aggregation performance. The aggregated model showed lower NMAE compared to the original model when clustering is based on a hybrid signal resolved at 60-minute time intervals, which is a combination of the electricity demand profile and AC modulation level.
\end{abstract}




\section{Introduction}

The building sector, given its large share in the total energy use, plays a central role in energy 72 policy. It accounts for about $40 \%$ of the total 73 energy use both in Europe and in the US (EIA 74 2017; EU 2010). Therefore, energy modeling for 75 demand forecasts or for assessing the impact 76 of energy management strategies in the build- 77 ing sector is of high importance. To this aim, 78 accurate simulation tools for large scale eval- 79 uations of the integrated supply-demand en- 80 ergy system are necessary. However, a proper 81 trade-off between the fidelity of representation 82 and the resultant computational effort has to 83 be found. Clustering techniques (Jain, Murty, 84 and Flynn 1999), which group similar data ob- 85 jects in the same cluster, can be used in order 86 to select representative buildings of the overall 87 building stock and use them to simplify its rep- 88 resentation. This paper presents and evaluates 89 such a methodology.

Several studies in the literature report the 91 use of clustering techniques for different appli- 92 cations. Nahmmacher et al. (2016), for exam- 93 ple, used clustering algorithms to select rep- 94 resentative days for long-term power systems 95 modeling. In this way, it is possible to select 96 a small number of days that adequately re- 97 flect the characteristic fluctuations of the re- 98 newable energy sources in the generation mix, 99 thus reducing the computational effort, while 100 maintaining the necessary diversity in tempo- 101 ral profiles. Buttitta, Turner, and Finn (2017) 102 applied a similar approach to define realistic 103 building occupant behavior, representative of 104 a large number of households, based on avail- 105 able survey data.

As far as the building sector is concerned, 107 often buildings are grouped on the basis of 108 their characteristics. Gao and Malkawi (2014) 109 showed the advantages of a multi-dimensional 110 clustering approach that enables energy bench- 111 marking among different types of buildings. 112 This was done by taking the most relevant 113 characteristics into account to define the build- ${ }_{114}$ ing energy performance. Jones, Lannon, and 115 Williams (2001) developed a method to group 116 buildings on the basis of some parameters 117 related to their energy performance: heated ${ }_{118}$ ground floor area, facade, window to wall ra- 119 tio, exposed end area and age. Santamouris 120 et al. (2007) applied intelligent fuzzy clustering techniques to classify school building energy data around clusters of similar characteristics. Moreover, Gaitani et al. (2010) proposed a clustering methodology based on principal components analysis to group school buildings in Greece and to define the typical building of each energy class by considering seven variables (heated surface, age of the building, insulation of the building, number of classrooms, number of students, school's operating hours per day, age of the heating system). The representative buildings can then be used to perform analysis on the potential energy savings for the specific group of school buildings.

Geyer, Schlüter, and Cisar (2017) considered a clustering method based on the sensitivity of buildings to retrofit strategies. In this way it is possible to effectively perform the retrofit of a large building stock by selecting the best retrofit measures, not only related to building age and type. Other studies, instead, tried to directly cluster the building load curves from time series data (Jota, Silva, and Jota 2011) to provide useful instruments to the energy manager to predict buildings loads and peak demand. For instance, Yang et al. (2017) proposed a clustering method based on k-shape algorithm to identify shape patterns in timeseries data, thus detecting building-energy usage patterns at different levels. The clustering result was further utilized to improve the accuracy of forecasting models. Yamaguchi, Shimoda, and Mizuno (2007) showed a district clustering modeling approach, where a district instead of a single building was considered as reference unit, in order to evaluate energy management at the city level. Iacovella et al. (2015) presented an algorithm for determining up to five representative appliances with artificial parameters to represent a larger set of thermostatically controlled loads.

The state of the art review highlights that clustering techniques in buildings can be used in different fashions and for different purposes. In each case, the starting point is represented by available data about building features or its behavior (e.g. energy performance...), grouped by means of techniques appropriate for the final knowledge purpose of the analysis. In this paper the main goal behind clustering of buildings is the representation of 
their energy flexibility in an efficient and effec- 173 tive way. As shown in previous work of the au- 174 thors (Patteeuw, Henze, and Helsen 2016), it is 175 very important to anticipate the electricity de- 176 mand of a building stock during flexible oper- 177 ation. The aim of this paper is to demonstrate 178 how the representative buildings of the clus- 179 ters can be used in aggregated simulation mod- 180 els maintaining the necessary accuracy. Given 181 the relevance of demand response (DR) pro- 182 grams to manage the electric energy demand 183 in buildings, there is a growing need for proper 184 models to simulate integrated energy systems, 185 where both the supply side and the demand 186 side and their interaction are represented with 187 sufficient detail (Patteeuw et al. 2015). In par- 188 ticular, Goy and Finn (2015) highlighted the 189 necessity to develop demand response estima- 190 tion tools at a large scale considering the build- 191 ings characteristics for electrically driven heat- 192 ing and cooling systems (i.e. heat pumps and 193 chillers).Other approaches, rather than cluster- 194 ing, have been used by different authors to rep- 195 resent the energy demand in integrated simula- 196 tions. E.g. Callaway (2009) uses a hybrid state 197 discrete time model to mimic thermostatic controlled loads (TLC) with a probability distribution of the TLCs population, while Hedegaard 198 et al. (2012) proposes a thermal building model add-on for the software Balmoral applied to the 199 building stock of existing individually heated 200 one-family houses in Denmark in 2030.

This paper presents, instead, the application 202 of a method called cluster-center-aggregation 203 (CCA) in building stock simulation and eval- 204 uates its performance. This CCA method is 205 based on clustering techniques for energy flexi- 206 bility evaluations in building stocks. The aim of 207 CCA is to reduce the overall building stock to a 208 number of representative buildings able to as- 209 sess, with sufficient accuracy, the total building 210 electric energy demand dynamics to be used in 211 integrated power system representations. The clustering algorithm is applied to electric power or AC staging data obtained by means of a ${ }^{212}$ simulation tool, written in Java, that repro- ${ }^{213}$ duces in detail all the buildings contained in ${ }_{214}$ a considered building stock. The total electric- ${ }_{215}$ ity demand profile from such a comprehensive ${ }_{216}$ simulation tool is then compared with the pre- ${ }_{217}$ diction of the aggregated demand side model ${ }_{218}^{217}$ that scales up the electricity demand of the ${ }_{219}$ representative buildings. The objective of this comparison is the determination of the proper number of representative buildings (i.e., number of clusters) in order to balance the opposing needs of reduced computational burden and loss of accuracy when assessing the demand flexibility of a building stock. The electric energy demand of buildings consists of deferrable loads, among them thermostatically controlled loads (e.g. cooling and heating by chillers, heat pumps or electric resistance), which can be shifted in time providing flexibility to the electric grid. The ability to represent and foresee such flexibility plays a crucial role in order to assess the demand shaping potential of the building stock. The proposed CCA methodology offers a simplified and reliable representation of thermostatically controlled electrical loads to be used in the evaluation of the economic and societal value of demand flexibility. In this piece of work the focus, and main novelty, lies in capturing the flexibility of the building stock by means of an aggregated demand side model obtained through clustering techniques.

\section{Methodology}

This section describes the CCA methodology and how its performance has been assessed. First, the general CCA methodology is described in Section 2.1) after which the specific application in the context of air-conditioning electricity demand flexibility is shown (Section 2.2 ). This methodology is compared to random sampling as the benchmark in Section 2.3 by means of the performance metrics introduced in Section 2.5. Section 2.4 presents the case study that is used to test the performance of cluster-based sampling in representing the building stock flexibility.

\subsection{General Cluster Center Aggregation (CCA)}

The goal of the Cluster Center Aggregation (CCA) is to draw a number of samples from a population and use these samples to represent the entire population. Figure 1 illustrates the basis steps of this CCA: cluster, center selection, and scale up. First, a number of fea- 

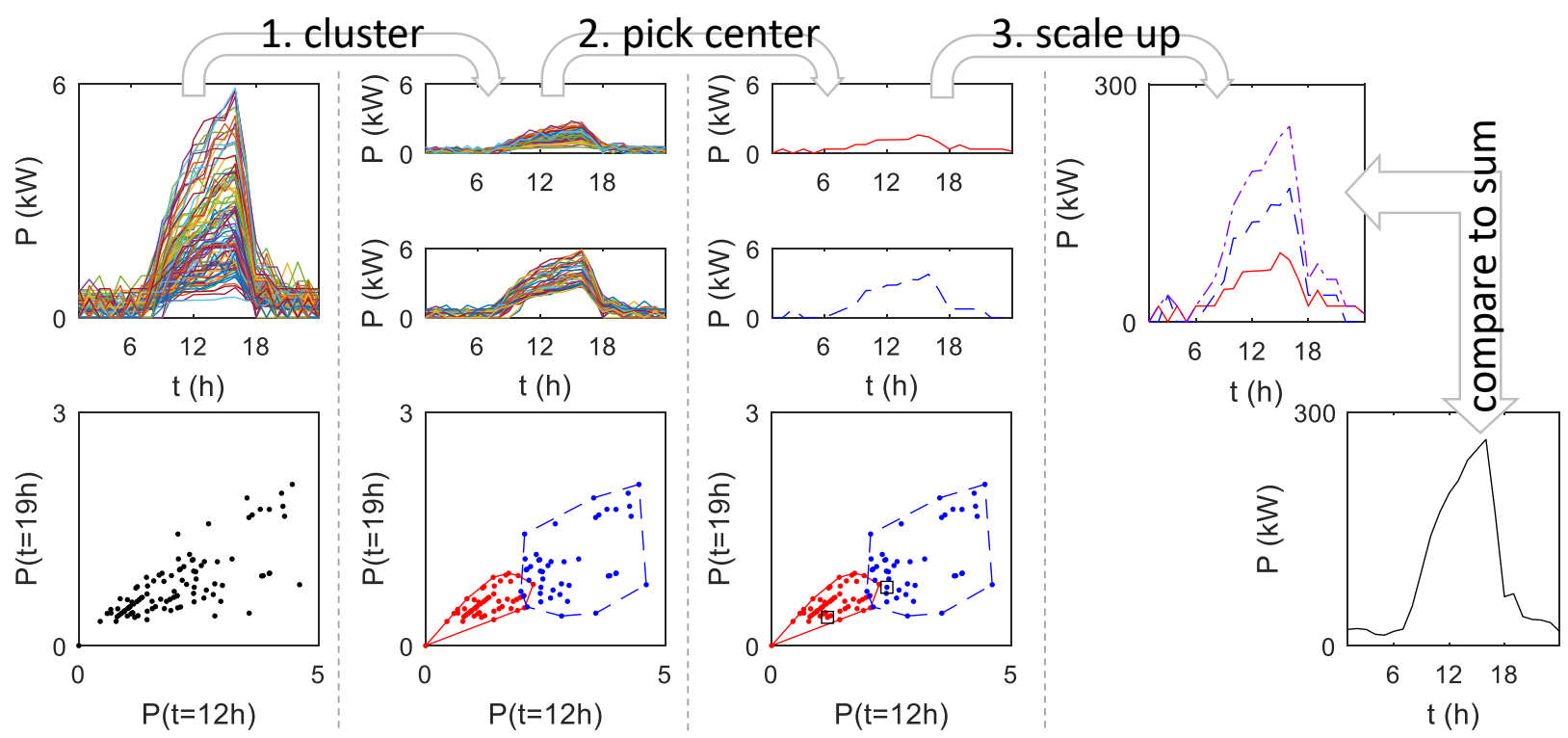

Figure 1.: Illustration of the CCA principle on 100 AC electricity demand profiles (top row). The clustering is performed for 24 time steps (i.e. 24 hours in a day) and hence in 24 dimensions. Since a 24 dimensional plot is not possible, only two of these time steps are illustrated (bottom row). In step 1, all profiles are clustered into two clusters. In step 2, the profile closest to the cluster centroid is selected as representative for that cluster. In step 3, each cluster's representative profile is scaled up with the number of profiles within that cluster. Last, it is evaluated how well the resulting electricity demand profile of these 3 steps (purple dash-dot line) compares to the sum of all 100 profiles (black line).

tures need to be selected from each member 243 of the population in order to group the mem- 244 bers of the population in clusters. In the second 245 step, the central member of the cluster is picked 246 up as a representative member of that cluster. 247 In the third step, the features of this selected 248 member are scaled up with the number of mem- 249 bers in that cluster, in an attempt to imitate 250 the features of the entire population. The main ${ }^{251}$ issue for application of the CCA method is to 252 justify whether the second and third steps are 253 allowed in a certain context or not. Throughout 254 this paper, the context of building flexibility is 255 considered.

\section{2. $C C A$ in air-conditioning electricity demand flexibility context}

The novelty of this paper lies in investigating the usefulness of the CCA methodology in the context of air-conditioning electricity demand flexibility. The population to start from is a large number of buildings. In this paper, this large number of buildings is modeled as de- scribed in Section 2.4 and is referred to as the 'full model' throughout this paper. This model consists of thousands of residential buildings equipped with central air-conditioning (AC) units and smart thermostats ${ }^{1}$. The aim of this paper is to attain an aggregated model which consists of representative buildings taken from the full model, whose AC electricity demand can be rescaled to replicate the full model electricity demand. Such an aggregated model can then be used e.g. in integrated representations of the electric power system to assess the flexibility potential of the building stock. Fig. 2 illustrates the CCA procedure in this context.

In this paper, the features upon which to perform the clustering are the measurement data of the buildings, taken from the output of the 'full model'. In the first step of CCA, this output data from the full model, called 'training data' throughout the rest of the pa-

\footnotetext{
${ }^{1}$ In this paper, a smart thermostat is defined as a thermostat which is connected to the internet in order to communicate indoor air temperature, set-point and the control signal it sends to the AC unit. Additionally, it is able to perform model predictive control in response to a price profile.
} 


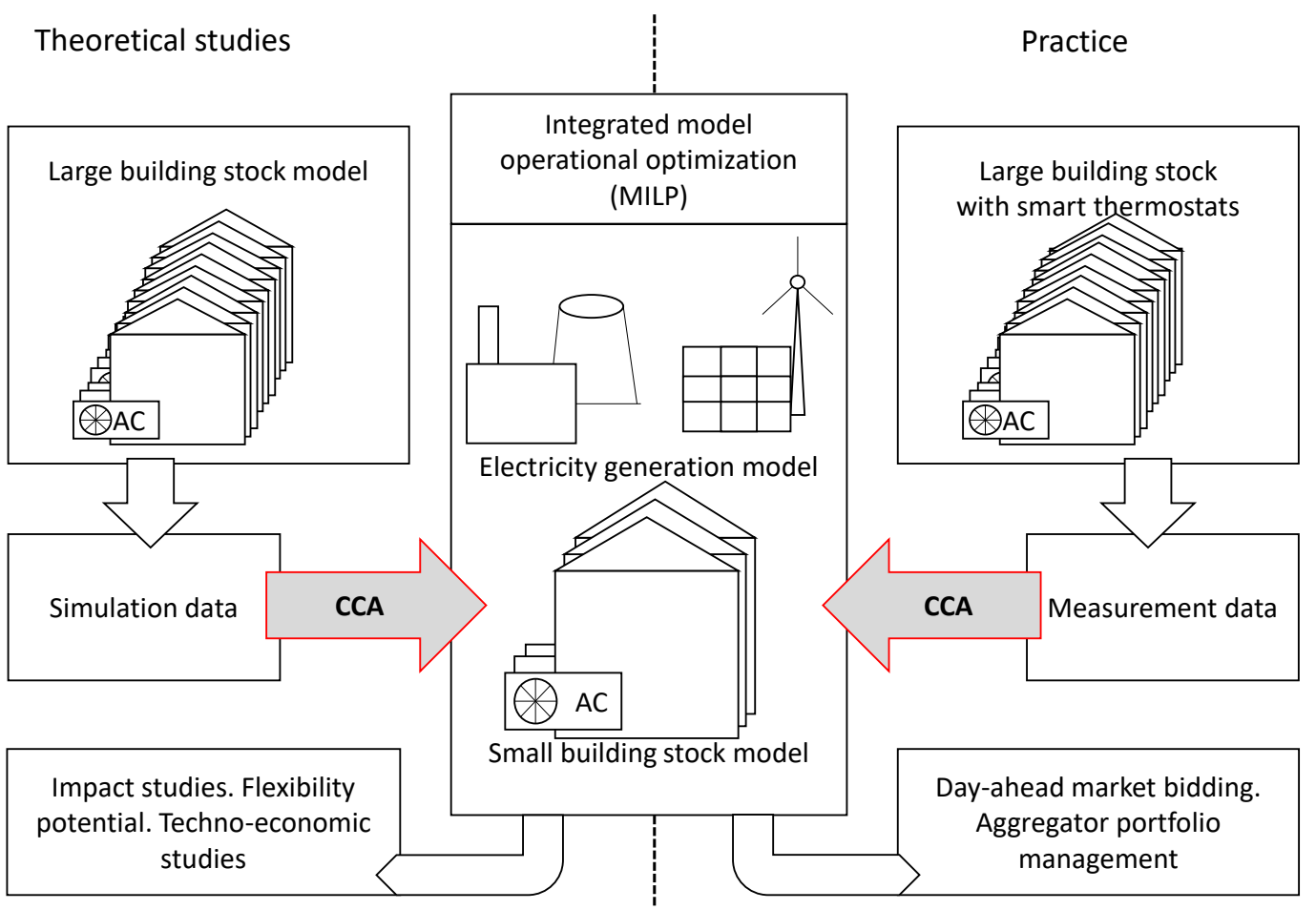

Figure 2.: Schematic representation of the use of CCA for demand response purposes in theoretical studies and in practice.

per, is taken and clustered. The data from the 290 full model mimics the data that could be avail- 291 able through the use of smart thermostats in 292 real applications in practice. In one case, this 293 smart thermostat could obtain direct measure- 294 ments of the 'electricity demand' (ED) profile 295 through a communication with the AC unit. 296 ED data is reported in $W$ and varies between 297 0 and the maximum power of the building. The 298 highest instantaneous electricity demand of one 299 building is $13500 \mathrm{~W}$ in this study.

If the communication with the $\mathrm{AC}$ unit is 301 not available, the smart thermostat only has 302 information on the control signals sent to the 303 AC unit. Since the control signals are typically 304 in the form of an on-off signal or staging sig- 305 nal, the smart thermostat only has information 306 of the modulation of the AC unit. This input 307 data is referred to in the remainder of the text 308 as 'AC modulation data' (ACMD). ACMD can 309 only take up the values 0,1 and 2 , e.g. the 310 stages of the AC unit.

In the case where both ED and ACMD data are available, a 'hybrid' data input can be defined. In this hybrid method, the input data is compiled of two sets of data: a period of ED and the ACMD of the same period. The
ACMD data is rescaled ${ }^{2}$ in order to achieve the same order of magnitude as the ED input data. Hence, both input data have equal importance in the clustering step.

Furthermore, this data could be fed to the clustering algorithm at 5-minute or 60-minute resolution. These two resolutions are the typical lowest and highest resolutions used for HVAC data. Intermediate resolutions are not studied in order to limit the number of cases in this paper. The 60-minute resolution is obtained by averaging the 5-minute resolution data, which largely filters out the on-off cycling of the AC unit.

The full model output data is summarized in a set of vectors: the ED or ACMD profile for each building, for a particular period in time (one week has been considered in this analysis). For the hybrid method, both data profiles of the same week are put adjacent to each other, so the input data profile is twice as long. A clustering algorithm then clusters these build-

\footnotetext{
${ }^{2}$ Based on Fig. 9, the ACMD data in this paper is multiplied by a factor of 10, while the ED data is expressed in $k W$. In this manner, both ED and ACMD input data are of the same order of magnitude.
} 


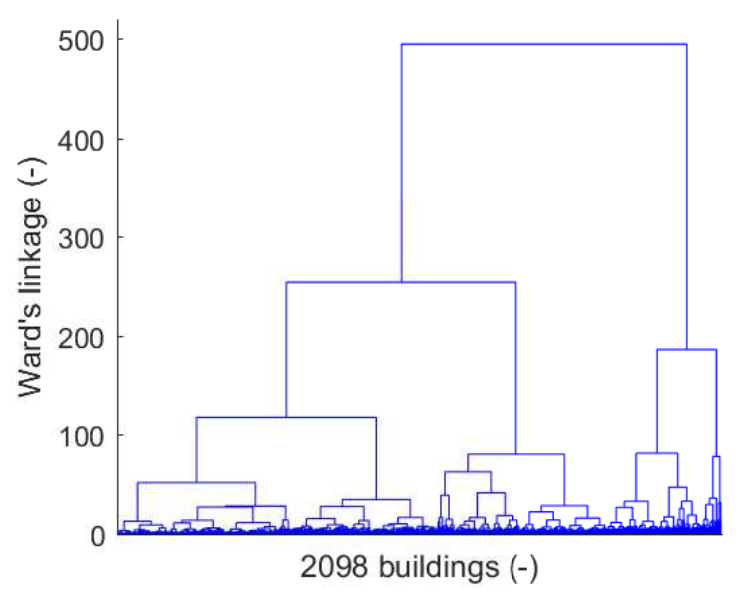

Figure 3.: Dendrogram of hierarchical clustering on ED data in $\mathrm{kW}$ and 60-minute resolution for the first week of July in Houston (see also Figure 5).

ings in groups of buildings with similar ED or 355 ACMD profiles. This similarity is based on the 356 Euclidean distance between the ED or ACMD 357 profiles. Hence, in this paper, the clustering 358 starts from data in multiple dimensions: the 359 number of time steps. This paper employs the 360 hierarchical clustering with Ward's minimum 361 variance method (Ward Jr 1963). There are nu- 362 merous clustering algorithms which could be 363 used in this context and comparing all of them 364 is out of the scope of this paper. Hierarchical 365 clustering is employed here since it leads to a 366 single reproducible result, as opposed e.g. to 367 $k$-means clustering where the random starting conditions influence the result. This hierarchical clustering is performed by the Matlab script clusterdata.m (MathWorks 2017a), using Euclidean distances and Ward's method for linkage. This linkage between two joined clusters $a$ and $b$ is calculated as the increase in $d(a, b)$, the ${ }^{368}$ total within-cluster sum of the squares of the ${ }^{369}$ distances between all objects in the cluster and ${ }^{370}$ the centroid of the cluster (MathWorks 2017b): ${ }^{371}$

$$
d(a, b)=\sqrt{\frac{2 n_{a} n_{b}}{n_{a}+n_{b}}}\left\|\bar{x}_{a}-\bar{x}_{b}\right\|_{2}
$$

with $n_{a}$ the number of elements in cluster $a, 374$ \|\|$_{2}$ the Euclidian distance and $\bar{x}_{a}$ the centroid 375 of cluster $a$. Fig. 3 shows an example of a result- 376 ing dendrogram using this clustering method. 377 The only remaining user-defined parameter for 378 the clustering is the number of clusters (i.e., the number of representative buildings for the aggregated model) to consider. This can be interpreted in Fig. 3 as 'cutting the cluster tree' at a certain value of Ward's linkage. The number of clusters is varied, and reported in a relative metric called sample size:

$$
\text { sample size }(\%)=\frac{\# \text { clusters } \cdot 100}{\# \text { buildings in full model }}
$$

In the second step of CCA, the clusters are translated to a set of representative buildings. For each cluster, the center is determined as the average profile of the ED or ACMD profiles. The building whose profile is closest to this center is selected as the representative building. This paper investigates the suitability of this building in representing its cluster, especially when different operational strategies are applied (e.g. DR programs). Applying this centering methodology for all clusters, yields a set of representative buildings, which is a sample from the total set of buildings.

In the third step of CCA, the electricity demand profiles of the representative buildings, $P^{r b}$, are rescaled. For each representative building of cluster $i$, the electricity demand profile $P_{i}^{r b}$ is multiplied by the number of buildings in the corresponding cluster, $N_{b, i}$. The resulting electricity demand profile of the aggregated model, $P^{C C A}$ is hence calculated as:

$$
P^{C C A}=\sum_{i=1}^{N_{c}} N_{b, i} P_{i}^{r b}
$$

with $N_{c}$ the number of clusters in this case.

Eventually such electricity profile of the aggregated model is used to represent the building stock in integrated models for demand response analysis, as illustrated in Fig 2.

\subsection{Random sampling as reference}

In this paper, random sampling is used as a benchmark for the aggregation performance. In the case of an aggregation with $N_{c}$ clusters and hence $N_{c}$ representative buildings, it is best to compare this to a random sampling 


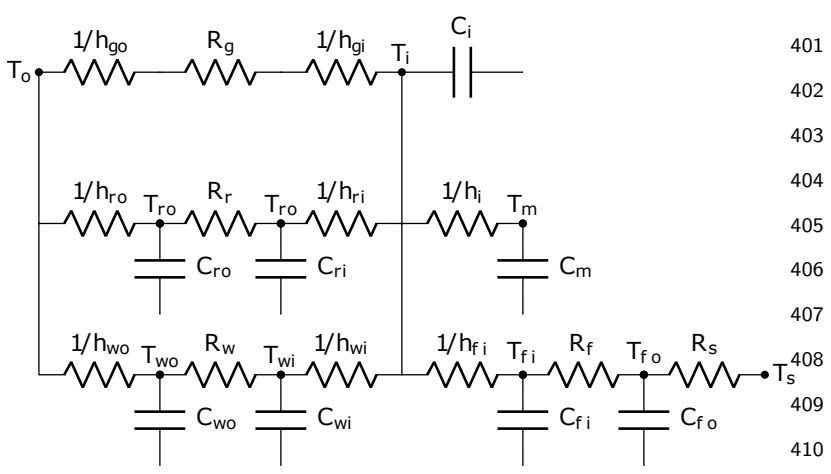

Figure 4.: The RC network representing the heat transfer in the reference building model (Corbin and Henze 2017a). The building is modeled as one thermal zone at a temperature $T_{i}$. The floor (f), external walls (w) and roof (r) are represented by two thermal capacities each, while the glazing $(\mathrm{g})$ has no thermal capacity. The internal walls and furniture are lumped within one 'thermal mass' capacity $(\mathrm{m})$. Heat exchange is present in the model with the outside air (o) and soil (s).

with also $N_{c}$ representative buildings. The aggregation based on random sampling consists of two steps. In a first step, $N_{c}$ random buildings are taken from the population. In a second step, the electricity demand profiles of each randomly chosen building $P_{i}^{\text {random }}$ is scaled up by an equal factor:

$$
P^{\text {aggr }, \text { random }}=\sum_{i=1}^{N_{c}} \frac{N_{p}}{N_{c}} \cdot P_{i}^{\text {random }}
$$
with $N_{p}$ the total number of buildings in the ${ }_{436}$ population. The random sampling is performed ${ }_{437}$ multiple times (for this study 20 times, see Sec- ${ }_{438}$ tion 3.3) in order to get an image of the spread ${ }_{439}$ of the performance of random sampling.

\subsection{Diverse Building Stock Case Study}

The performance of the CCA methodology is 445 tested on output data of a building stock model 446 that simulates the cooling demand of a large ${ }^{447}$ number of US buildings. This detailed dynamic ${ }^{448}$ simulation model was developed by Corbin and 449 Henze (Corbin and Henze 2017a,b) and val- 450 idated with respect to BESTEST-EX (Jud- ${ }^{451}$ koff et al. 2010) by Corbin (Corbin 2014). The ${ }^{452}$ model comprises three cases of building stocks in different US climate zones: Houston (Texas), New York (New York) and Los Angeles (California). The Texas case consists of 2146 buildings from which 2098 are equipped with AC. For New York this is 1506 (1114 with AC) and for Los Angeles this is 1326 (711 with AC). In this study, the non-HVAC electricity demand, which is also an output from the model, is not taken into account. Each of the buildings differ in type (mobile, detached or appartment), floor area, cooling set point and building integrity. This results in different parameters for the insulation of the roof, walls, floor and windows as well as a different infiltration rate and thermal mass. For each climate zone separately, these values are randomly sampled from the Residential Energy Consumption Survey (U.S. Energy Information Administration 2009) data that was collected and made available by the US Energy Information Administration. This results in different thermal properties of the buildings depending on the climatic zone, as illustrated in Table 1.

Each building is modeled as one thermal zone. The heat transfer in the building is modeled through a network of thermal resistances and capacities as illustrated in Fig. 4 for which all $\mathrm{R}$ and $\mathrm{C}$ values are constant. The only exceptions to this are the exterior film coefficients that depend on the wind speed. The solar heat gains are based on Liu and Jordan (1960) for an isotropic clear sky. The buildings are equipped with a central air conditioning. The main component of the $\mathrm{AC}$ is the single and dual stage electric direct expansion air cooling coil (UIUC and LBNL 2005). This cooling coil is complemented with a constant volume fan. The building modeling was validated with BESTEST-EX (Judkoff, Neymark, and Polly 2011). The internal heat gains from occupants are based on a relaxed seated person while the gain from appliances are modeled based on nominal energy demand, schedules and sensible heat fraction (Corbin 2014). The temperature set points vary among the different buildings but are constant during the day. The AC control of each building is performed independent of the other buildings, by means of a dual mode thermostat with a hysteresis of $0.5 \mathrm{~K}$. In addition, each building is assumed to have a model predictive controller 
Table 1.: Home characteristics for each climatic zone selected for study, taken from Corbin and Henze (2017a)

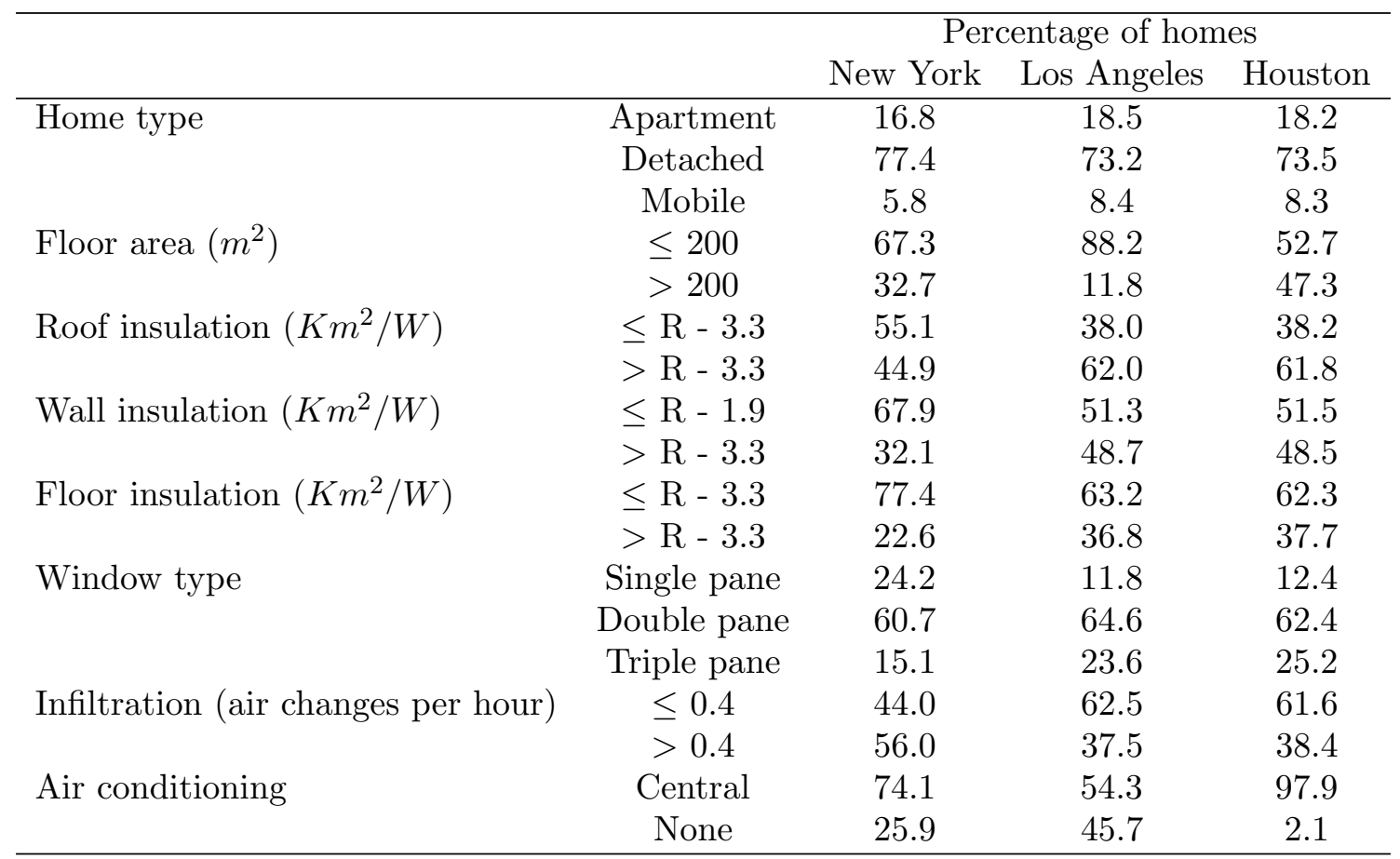

(MPC) that uses a particle swarm optimization 479 (PSO) (Corbin 2014) based on the canonical formulation of Eberhart and Kennedy (1995). This formulation is enhanced with a taboo list for previous candidates and familiar box constraints. The increment is 0.1 and a maximum velocity is 0.25 . The MPC can alter the upper and lower bound for the hysteresis controller between certain predefined limits. A fully detailed description of the reference model can be found in Corbin (2014).

Fig. 5 shows some typical input and output data of the building stock model for Houston. The first two figures show the solar heat gains, ambient air temperature and wind speed. These weather input conditions are used for all buildings in the Houston model. The last two figures show the output of the model for ${ }^{490}$ one building (left axis, blue) and for all build- ${ }^{491}$ ings with AC (right axis, orange). What can ${ }^{492}$ be observed first is the strong cycling of the ${ }^{493}$ $\mathrm{AC}$, both in the indoor temperature and in the electricity demand. When considering the 2098 buildings with AC in Texas, the electricity demand shows a strong correlation with the solar gains and outdoor temperature.

\subsection{Sampling Performance Evaluation}

The interest of this paper lies in the performance assessment of demand flexibility and, in particular, how well normal operation data suits the aggregation methodology in predicting flexible building operation in response to DR and demand shaping signals. The aggregated electricity demand profile should match the full electricity demand profile as accurately as possible, evaluated by the mean absolute error (MAE) over $h$ time steps with index $j$ :

$$
M A E=\frac{\sum_{j=1}^{h}\left|P_{j}^{f u l l}-P_{j}^{C C A}\right|}{h} .
$$

The MAE is normalized by the mean value $\mu$ of the full electricity demand profile in order to have a metric comparable for the different considered case studies:

$$
N M A E=M A E / \mu=\frac{\sum_{j=1}^{h}\left|P_{j}^{f u l l}-P_{j}^{C C A}\right|}{h \cdot \frac{\sum_{j=1}^{h} P_{j}^{f u l l}}{h}} .
$$

regime, all buildings within one case are sub- 

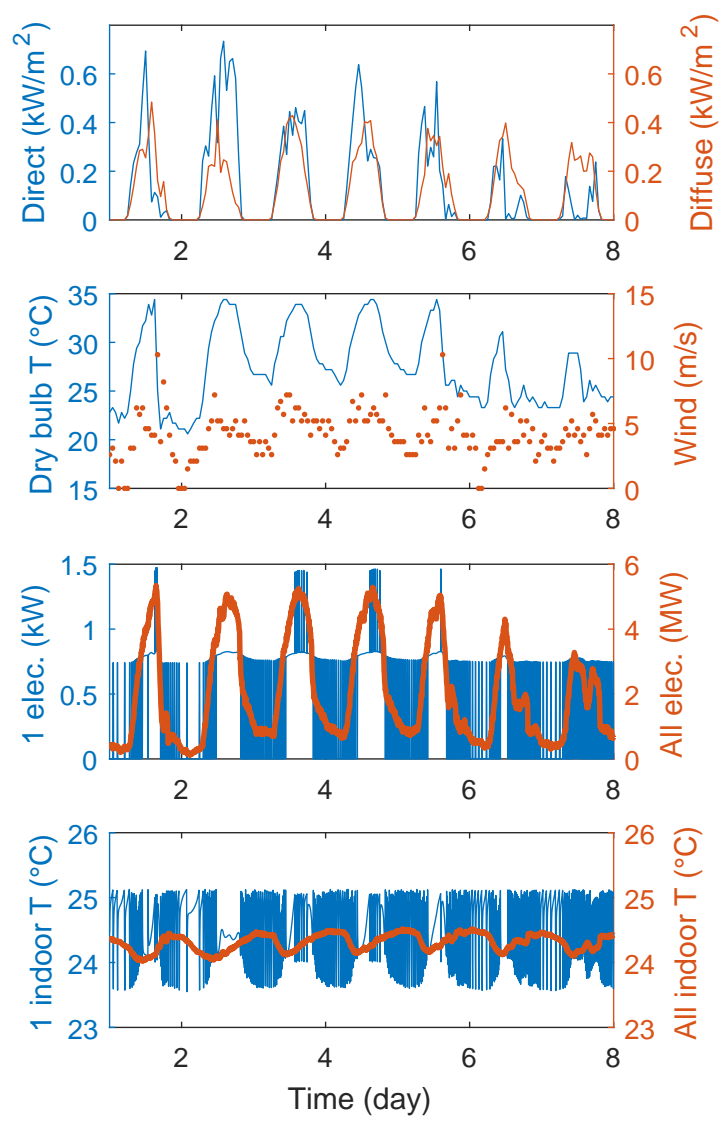

Figure 5.: Data for the first week of July ${ }^{519}$ in Houston, Texas. From top to bottom: di- ${ }^{520}$ rect (left) and diffuse (right) solar heat gains; outside dry bulb temperature (left) and wind speed (right); HVAC electricity demand of one building (left) and all buildings combined (right); indoor air temperature of one building (left) and all buildings combined (right).
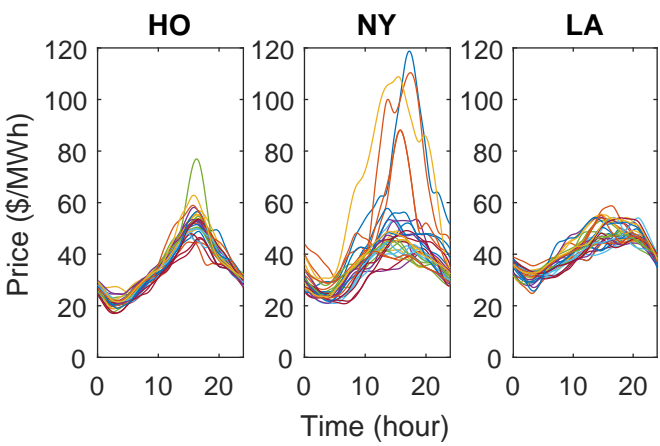

Figure 6.: Electricity price profiles for every day of July for the cases of Houston (HO), New York (NY) and Los Angeles (LA). 0 jective function becomes

$$
\min \sum_{j=1}^{H} e_{j} \cdot P_{j}^{H V A C} .
$$

The electricity price profiles shown in Fig. 6 are used for each day of the month. These profiles are based on the wholesale market prices corresponding to the region in which the cities are located. Based on historical wholesale market prices, Corbin (2014) determined these price profiles for the typical weather file used throughout the model. In the scenario of price responsive operation, the temperature bounds for comfort are relaxed to allow for a stronger response to the price profile. Before 8 a.m. and after 6 p.m., the lower bound on the indoor temperature is lowered by $2 K$ in order to allow for precooling. During absence of the occupants, between 8 a.m. and 6 p.m., the upper bound of the indoor temperature is increased by $3 K$ while the lower bound is decreased by $5 K$. Hence, in this period of absence there is 
a significant potential for load shifting. The plots 'normal' and 'price' in Fig. 7 show that the combination of the variable price profile and the wide band on temperature set-point can lead to extreme electricity demand profiles. The results for the 'price' scenario are hence for a fairly extreme flexibility scenario.

\section{Results}

In Section 3.1 the results of the application of the CCA methodology are illustrated. In particular, the impact of the choice of input data (i.e. sampling time and type of signal) on the performance of CCA is analyzed (Section 3.2). Finally, the performance of CCA is compared to a random sampling approach in Section 3.3.

The evaluation of the CCA methodology is common in the context of unsupervised machine learning. Measurements for a couple of days are used as training data for CCA (see Fig. 7 (top)). The goodness of fit (NMAE in this study) of the resulting representative buildings and their factors $N_{c}$ are tested on this training data. But more importantly, the goodness of fit is validated on validation data, which is data stemming from the same system but for a different time period (see Fig. 7 (bottom)).

\subsection{CCA Methodology Application}

Fig. 7 illustrates the application and perfor- 588 mance of the CCA. The aim of the aggrega- 589 tion is to predict the electricity demand pro- 590 file of the full building stock $P^{\text {full }}$ ('Full' in ${ }^{591}$ Fig. 7). The CCA aggregation methodology re- 592 constructs the electricity demand profile with a limited number of representative buildings $P^{C C A}$. The model is trained in normal operation for a summer week in July and then tested 594 for the whole month. The results are shown for 595 multiple numbers of representative buildings, 596 normalized to a sample size expressed in per- 597 centage (Eq. 2). As can be seen in Fig. 7, the 598 performance of the aggregation on the training 599 data is very good, even for a small sample size 600 of $1 \%$. Also, in the case of testing with a flat 601 electricity price ('Normal' case in Fig. 7), the 602 aggregated representation performs well. Yet, 603 when price responsive MPC is applied in order 604 to assess the flexible behavior as explained in 605
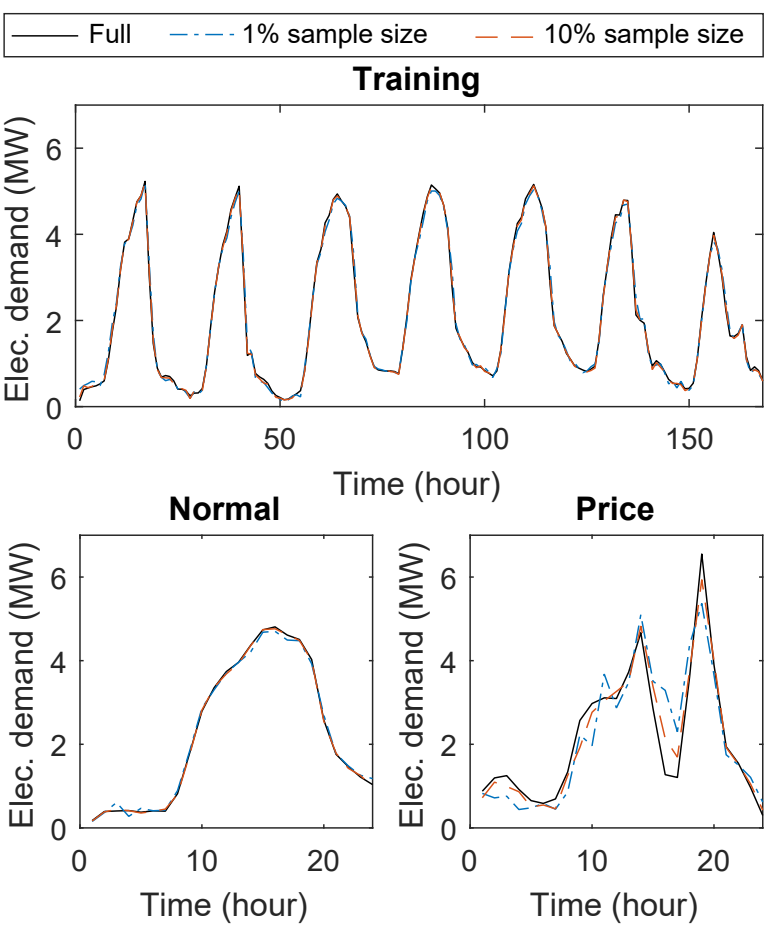

Figure 7.: Reconstruction of the electricity demand profile for Houston, Texas. Clustering based on electricity demand data for a week in hourly resolution. Aggregation performance illustrated for the training data of a week. The performance is also shown for July 19th, for both normal operation and for responsive price for both a $1 \%$ and $10 \%$ sample size of buildings.

Section 2.5 ('Price' case in Fig. 7), the MAE rises and it is evident that better performance is achieved by increasing the sample size. In this case, a sample size of $1 \%$ gives a MAE in predicting the electricity demand of $0.50 \mathrm{MW}$. This reduces to $0.23 \mathrm{MW}$ for a sample size of $10 \%$.

\subsection{Choice of Input Data}

This section investigates the importance of the choice and pre-processing of the input data used for clustering, as introduced in Section 2.2. The data could either consist of direct measurements of electricity demand (ED) or in the absence of these, AC modulation data (ACMD). This data could be attained in 5 or 60-minute resolution. For these four input preprocessing options, Fig. 8 illustrates the normalized mean absolute error (NMAE) between the estimated and actual feeder electricity demand for multiple cases (Houston (HO), New 
York (NY) and Los Angeles (LA)). Moreover, 626 both normal operation with a flat price and the 627 price responsive profiles are considered. The ${ }_{628}$ latter is used to assess the performance for flex- 629 ible operation.
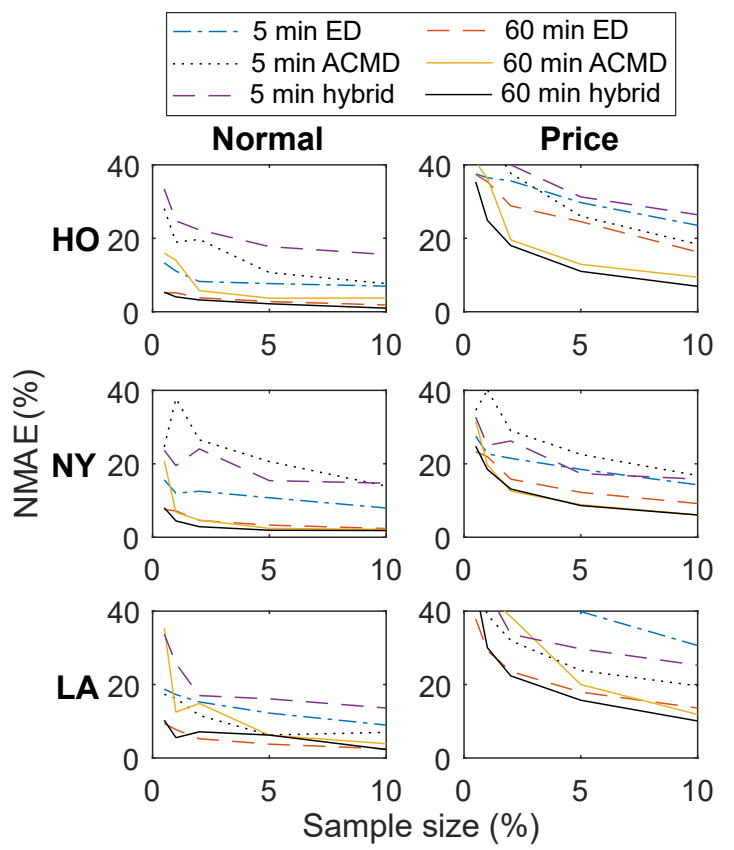

630 631 632 633 634 635 636 637 638 639 640

Figure 8.: Normalized mean absolute error 651 (NMAE) as a function of the sample size, de- 652 termined for normal operation and operation 653 under price incentives (Fig. 6) for the month 654 of July for Houston (HO), New York (NY) and 655 Los Angeles (LA). Comparison of six cluster- 656 ing options, based on clustering of profiles in 657 five minute $(5 \mathrm{~min})$ or 60 -minute $(60 \mathrm{~min})$ res- 658 olution of electricity demand (ED), AC modu- 659 lation data (ACMD) or both ED and ACMD 660 (hybrid).

Regarding the resolution of input data, Fig. 8663 shows that providing the clustering method- 664 ology with input data in 60-minute resolu- 665 tion generally outperforms 5-minute resolution 666 data. The shorter resolution still contains the ${ }^{667}$ AC cycling artefacts, which appears to ham- 668 per the clustering in finding good representa- 669 tive buildings.

Regarding the type of input data, no clear ${ }^{671}$ preference between using directly electricity de- 672 mand data ('ED' in Fig. 8) or AC modulation ${ }^{673}$ data ('ACMD' in Fig. 8) emerges. The former 674 performs the best in the scenario of a flat elec- 675 tricity price. In this scenario, the available de- 676 mand flexibility of the buildings is not utilized 677 and all buildings follow their regular cooling schedule. Using electricity demand measurements as input data for the clustering performs well in this scenario, leading to a NMAE below $5 \%$. Clustering focusses strongly on the absolute value of the input profiles and hence a good representation is attained. When the flexibility is activated with a price profile, ACMD outperforms ED and shows lower errors for most cases. What emerges from this analysis is that focussing on the modulation of the air conditioning captures the demand flexibility better.

Generally speaking, a variable electricity price appears to have a significant impact on aggregation performance. When there is no electricity price incentive ('Normal' in Fig. 8), the NMAE stays easily below $10 \%$ of the mean electricity demand. With electricity price incentives ('Price' in Fig. 8), the NMAE is higher and quickly rises to $20 \%$ of the mean electricity demand. From this, it appears that flexible operation is harder to capture for aggregation.

The use of electricity demand data ('ED') delivers better results for normal operation while AC modulation data ('ACMD') delivers better results for flexible operation. The resulting clusters from both methods with a sample size of $1 \%$ for Houston (21 samples, 60-minute resolution) are illustrated in Fig. 9. Observing the cluster arrangement, it can be seen how clustering based on ED ignores the AC modulation level contained in the ACMD profile (Fig. 9a), while clustering based on ACMD ignores the information contained in the ED profile (Fig. 9b).

As introduced in the Section 2.2, the 'hybrid' method uses both ED and ACMD data. Figure 9c shows how clustering on both data simultaneously leads to fairly different clusters. The performance of the hybrid method is assessed in Fig. 8. The hybrid method based on 5 -minute resolution data shows equally poor performance as the other 5 -minute data types. However, the hybrid method with data at 60minute resolution, clearly outperforms ED and ACMD in most cases. For normal operation, it gets close or slightly improves upon using ED data. For flexible operation, it outperforms both ED and ACMD, sometimes by a significant margin.

The results in Fig. 8 are shown for an entire month. There appeared to be no clear cor- 

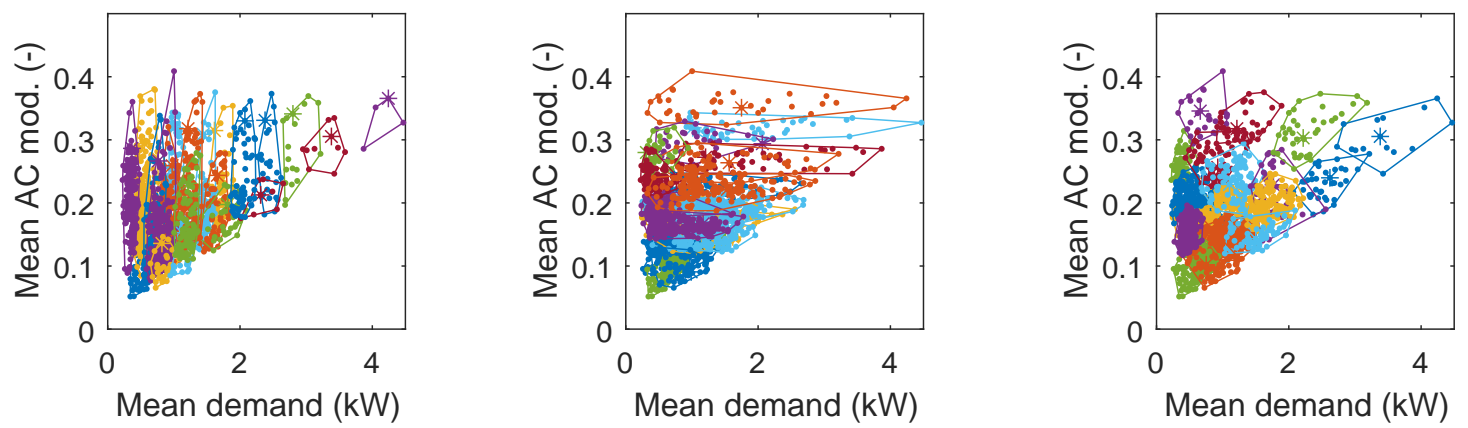

(a) Clustering based on electric(b) Clustering based on AC mod- (c) Clustering based on electric power ulation

power and $\mathrm{AC}$ modulation

Figure 9.: Illustration of resulting clusters based on electricity demand as input (Fig. 9a), AC modulation data as input (Fig. 9b) and a mix of both input data (Fig. 9c) which is coined 'hybrid' method. The clusters are illustrated by showing the mean electricity demand over the period on the $x$-axis and the mean AC modulation data (called 'mean AC mod.') on the $y$-axis. Buildings in the same cluster have the same color and are also surrounded by a convex hull for clarity.

relation between the NMAE per day and the variance of the price profile for the corresponding day. In other words, there is no significant difference in aggregation performance for days with either a strong or a weak price incentive.

The CCA methodology was repeated with input data stemming from multiple priceresponsive days. The result from this repetition are generally in line with using normal operation days as input, as shown in Fig. 8. Hence, the results from this variation are not shown separately.

Finally, the performance of the CCA methodology was also tested for normal operation during the month of May. In other words, representative buildings which were chosen based on data in July were tested for normal operation measurements in May. The results are very similar to Figure 8 and are hence not repeated here. From this, it appears that the CCA methodology can also be used to predict the full electricity demand profile during periods of lower cooling demand.

\subsection{Comparison to Random Sampling}

As described in Section 2.3, the results are com- 709 pared to the benchmark of random sampling. 710 Fig. 10 compares this random sampling to 711 the best performing CCA method, using hy- 712 brid data in 60-minute resolution. What can 713 be noted first is the excellent performance of 714 CCA compared to random sampling in case of 715
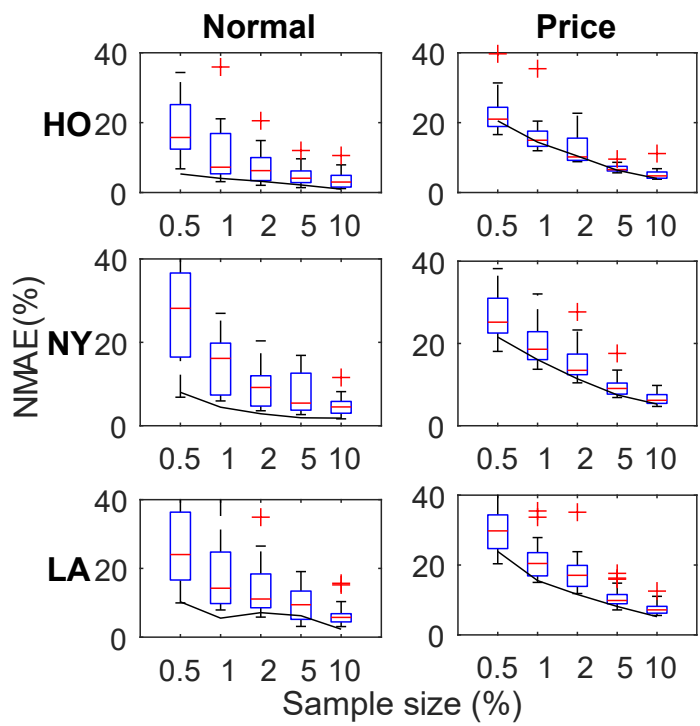

Figure 10.: Second evaluation of the normalized mean absolute error (NMAE) normalized by the mean of the full electricity demand as a function of the sample size. The full line shows the results for the aggregation based on the hybrid case in 60-minute resolution. The box plots show the results for random sampling, repeated 20 times. The red plus signs show the outliers of the box plot.

a flat price profile. The NMAE of CCA is in most cases smaller than $90 \%$ of the random sampling results. This illustrates how well CCA performs in predicting the normal operation of a large set of buildings.

In the scenario of flexible operation ('Price' in Fig. 10), it can be seen that CCA outper- 
forms random sampling less drastically. Over- 765 all, CCA outperforms 60 to $90 \%$ of the ran- 766 dom sampling results. For Houston, CCA out- 767 performs around $60 \%$ of the random sampling 768 cases. For New York and Los Angeles, CCA is 769 even better, outperforming 75 to $90 \%$ of the 770 random sampling results.

The advantage of CCA in this context is that 772 it directly leads to a single set of representative 773 buildings that do a fairly good job at capturing 774 the flexibility of the population of buildings. 775 This cannot be said from the random sampling, 776 which on average shows good performance but 777 with a wide spread in performance. For exam- 778 ple, taking a sample of $0.5 \%$ buildings in the 779 New York case with price incentive, could lead 780 to a NMAE between 18 and $39 \%$ compared to 781 the average electricity demand during that day. 782

\section{Discussion}

As far as the sample size (i.e., number of rep- ${ }^{787}$ resentative buildings) is concerned, the sample ${ }^{788}$ size quickly needs to be $10 \%$ of the population ${ }^{789}$ size in this paper in order to obtain good re- 790 sults in responsive price profiles, as can be seen 791 in Figs. 7 and 8. A sample size of $10 \%$ is fairly ${ }^{792}$ large and represents a complexity reduction of 793 only a factor 10 . Although it must be noted ${ }^{794}$ that in this paper, the population sizes of build- 795 ings with AC are pretty low: 2098 for Texas, 796 1114 for New York and 711 for Los Angeles. 797 Given a limited population size, this automati- 798 cally leads to a high relative sample size needed 799 (Krejcie and Morgan 1970). For example, for a 800 confidence level of $95 \%$, a margin of error of $5 \% 801$ and a population size of 2098, a classical sample ${ }^{802}$ size calculation (Krejcie and Morgan 1970) ad- 803 vises a sample size of 325 or $15 \%$. If we increase the population size to $1,000,000$ buildings, this sample size calculator advises 384 samples or 804 $0.00038 \%$. Hence, if the aggregation methodology presented in this paper is used on larger 805 population sizes, it can be expected that the 806 relative complexity reduction will be larger. $\quad 807$

When the aggregation is based on AC modu- 808 lation data, the performance in terms of NMAE 809 in flexible operation approaches that of CCA 810 with hybrid data (see Fig. 8). This is an in- 811 teresting result in the context of smart ther- 812 mostats. As many smart thermostats do not 813 have ED data available, they can only rely upon AC modulation data. In this context, they could perform the CCA methodology in order to attain a representative set of buildings. In practice, specifically only these buildings could be equipped with smart meters. The measurements of these smart meters can then be scaled up in order to get a good estimate of the full building population's electricity demand during flexible operation. However, care should be taken that the absence of a smart meter in a building is not correlated to certain building characteristics, which could create a bias in the sampling. Note that in this study, we use data stemming from a simulation model. If measurement data is used as input for the CCA method, this data should be reliable by avoiding wrong or absent measurements. A check for corrupt measurement data is thus needed, after which this data should be removed from the data set to be used in the CCA method.

Furthermore, this paper illustrates that applying CCA leads to an error and hence an uncertainty on a building stock's electricity demand profile. Such clustering can be used in DR programs (Iacovella et al. 2015). Bruninx et al. (2017) showed that a large uncertainty on the building stock's electricity demand can limit its perceived controllability and hence lower the value of DR for a system operator. Hence, when combining the representative building models with an electricity generation model in an integrated modeling framework (Patteeuw et al. 2015), an appropriate sample size needs to be chosen. A too small sample size will lead to large uncertainty on the building stock's electricity demand and make the DR scheduling unreliable. A too large sample size will lead to impractical calculation times.

\section{Conclusion}

The main novelty of this paper is the application of a cluster-center-aggregation (CCA) methodology in representing the flexibility of a diverse building stock AC electricity demand. Hierarchical clustering is used to group buildings on the basis of their electricity demand (ED) or AC modulation data (ACMD) for the electrical cooling system. For every cluster, the building with the electric energy demand pro- 
file closest to the average ED or ACMD profile 863 of that cluster is selected as a representative 864 building.

The model has been tested on three cases 866 composed of buildings stocks in different cities 867 and climate zones (Houston, New York or Los 868 Angeles) with 2098, 1114 or 711 modeled build- 869 ings respectively. The proposed model is in- 870 tended for the estimation of demand flexibility provided by the electricity for thermostatically controllable loads, such as central AC systems and heat pumps for cooling and heating. In order to represent the flexible operation, different price profiles have been considered. Results ${ }_{873}^{872}$ show the crucial role of data pre-processing to ${ }_{874}^{873}$ obtain low NMAE values for estimating the full ${ }_{875}^{874}$ building stock electricity demand profile. An 876 appropriate time resolution for input data is 877 60-minutes, with the use of ED showing better 878 results for normal operation and, conversely, 879 ACMD for flexible operation. Combining both 880 signals (ED and ACMD) outperforms all the ${ }^{881}$ other clustering options when a dynamic price ${ }^{882}$ profile is considered. When electricity demand ${ }^{883}$ profiles are not available, AC modulation data ${ }^{884}$ at 60-minute resolution still performs well dur- ${ }^{885}$ ing flexible operation, which can be useful for ${ }_{887}^{886}$ smart thermostats that do not have electric de- ${ }_{888}^{88}$ mand information at their disposal. Finally, the ${ }_{889}$ electricity demand profile is harder to estimate 890 during flexible operation, since the NMAE is 891 higher in all studied cases. For example for hy- 892 brid data (a mix between ED and ACMD data) 893 in 60-minute resolution, the NMAE is typically ${ }^{894}$ $5 \%$ during normal operation for a sample size ${ }^{895}$ of $1 \%$. In other words, the model size can be ${ }^{896}$ reduced with a factor 100 with only a NMAE ${ }^{897}$ of $5 \%$. For flexible operation, the NMAE is typ- ${ }_{898}^{898}$ ically $10 \%$ at a sample size of 5 to $10 \%$, hence ${ }_{900}^{899}$ for a model size reduction of 10 to 20 .

\section{Acknowledgements}

This study is part of the research project 'To- ${ }^{906}$ wards a sustainable energy supply in cities'. ${ }^{907}$ This project receives the support of the Eu- ${ }^{908}$ ropean Union, the European Regional Development Fund ERDF, Flanders Innovation \& ${ }_{911}$ Entrepreneurship and the Province of Lim- 912 burg. Furthermore, Dieter Patteeuw gratefully 913 acknowledges the Flemish research foundation 914
FWO for the grant for a long stay abroad at the University of Colorado Boulder. The authors also thank Kenneth Bruninx for his advise on clustering and Ján Drgoňa for his advise on modulation modeling. The computational resources and services used in this work were provided by the Hercules Foundation and the Flemish Government- department EWI.

\section{References}

Bruninx, Kenneth, Yury Dvorkin, Erik Delarue, William D'haeseleer, and Daniel S Kirschen. 2017. "Valuing Demand Response Controllability via Chance Constrained Programming." IEEE Transactions on Sustainable Energy. In print .

Buttitta, Giuseppina, William Turner, and Donal Finn. 2017. "Clustering of Household Occupancy Profiles for Archetype Building Models." Energy Procedia 111: 161 - 170. 8th International Conference on Sustainability in Energy and Buildings, SEB-16, 11-13 September 2016, Turin, Italy. Callaway, Duncan S. 2009. "Tapping the energy storage potential in electric loads to deliver load following and regulation, with application to wind energy." Energy Conversion and Management 50 (5): 1389-1400.

Corbin, CD, and GP Henze. 2017a. "Predictive control of residential HVAC and its impact on the grid. Part I: simulation framework and models." Journal of Building Performance Simulation 10 (3): 294-312.

Corbin, CD, and GP Henze. 2017b. "Predictive control of residential HVAC and its impact on the grid. Part II: simulation studies of residential HVAC as a supply following resource." Journal of Building Performance Simulation 10 (4): 365377.

Corbin, Charles D. 2014. "Assessing Impact of Large-Scale Distributed Residential HVAC Control Optimization on Electricity Grid Operation and Renewable Energy Integration." PhD diss., University of Colorado, CO, U.S.A.

Eberhart, Russell, and James Kennedy. 1995. "A new optimizer using particle swarm theory." In Micro Machine and Human Science, 1995. MHS'95., Proceedings of the Sixth International Symposium on, 39-43. IEEE.

EIA. 2017. "How much energy is consumed in residential and commercial buildings in the United States?" Accessed: September 2017, http://www.eia.gov/tools/faqs/faq.cfm? id $=86 \& t=1$.

EU. 2010. "DIRECTIVE 2010/31/EU OF THE 
EUROPEAN PARLIAMENT AND OF THE 971 COUNCIL of 19 May 2010 on the energy 972 performance of buildings." Accessed: Septem- 973 ber 2017, https://ec.europa.eu/energy/en/ 974 topics/energy-efficiency/buildings. $\quad 975$ Gaitani, N., C. Lehmann, M. Santamouris, G. Mi- 976 halakakou, and P. Patargias. 2010. "Using princi- 977 pal component and cluster analysis in the heating 978 evaluation of the school building sector." Applied 979 Energy 87 (6): 2079 - 2086.

Gao, Xuefeng, and Ali Malkawi. 2014. "A new 981 methodology for building energy performance 982 benchmarking: An approach based on intelligent 983 clustering algorithm." Energy and Buildings 84: 984 $607-616$

985

Geyer, Philipp, Arno Schlüter, and Sasha Cisar. 986 2017. "Application of clustering for the devel- 987 opment of retrofit strategies for large building 988 stocks." Advanced Engineering Informatics 31: 989 $32-47$.

990

Goy, Solène, and Donal Finn. 2015. "Estimating 991 Demand Response Potential in Building Clus- 992 ters." Energy Procedia 78: 3391 - 3396. 6th In- 993 ternational Building Physics Conference, IBPC 994 2015.

995

Hedegaard, Karsten, Brian Vad Mathiesen, Henrik 996 Lund, and Per Heiselberg. 2012. "Wind power in- 997 tegration using individual heat pumps - Analysis 998 of different heat storage options." Energy 47 (1): 999 284-293.

1000

Iacovella, Sandro, Frederik Ruelens, Pieter Vinger- 1001 hoets, Bert Claessens, and Geert Deconinck. 1002 2015. "Cluster control of heterogeneous thermo- 1003 statically controlled loads using tracer devices." 1004 IEEE Transactions on Smart Grid 8 (2): 528-1005 536.

1006

Jain, Anil K, M Narasimha Murty, and Patrick J 1007 Flynn. 1999. "Data clustering: a review." $A C M_{1008}$ computing surveys (CSUR) 31 (3): 264-323. 1009

Jones, P. J., S. Lannon, and J. Williams. 2001.1010 "Modeling building energy use at urban scale." 1011 In Proceedings of Building Simulation, 175 - 180. 1012 Jota, Patricia R.S., Valéria R.B. Silva, and Fábio G. 1013 Jota. 2011. "Building load management using 1014 cluster and statistical analyses." International 1015 Journal of Electrical Power and Energy Systems 1016 33 (8): $1498-1505$.

1017

Judkoff, Ron, Joel Neymark, and Ben Polly. 2011.1018 Building Energy Simulation Test for Existing 1019 Homes (BESTEST-EX). National Renewable 1020 Energy Laboratory.

1021

Judkoff, Ron, Ben Polly, Marcus Bianchi, and Joel 1022 Neymark. 2010. Building energy simulation test 1023 for existing homes (BESTEST-EX); Phase 11024 Test Procedure: Building Thermal Fabric Cases. 1025 Technical report. National Renewable Energy 1026
Lab.(NREL), Golden, CO (United States).

Krejcie, Robert V, and Daryle W Morgan. 1970. "Determining sample size for research activities." Educational and psychological measurement 30 (3): 607-610.

Liu, Benjamin YH, and Richard C Jordan. 1960. "The interrelationship and characteristic distribution of direct, diffuse and total solar radiation." Solar energy 4 (3): 1-19.

MathWorks. 2017a. "Clusterdata. Agglomerative clusters from data." Accessed: September 2017, https://mathworks.com/help/stats/ clusterdata.html.

MathWorks. 2017b. "Linkage. Agglomerative hierarchical cluster tree." Accessed: October 2017, https://mathworks.com/help/stats/ linkage.html.

Nahmmacher, Paul, Eva Schmid, Lion Hirth, and Brigitte Knopf. 2016. "Carpe diem: A novel approach to select representative days for long-term power system modeling." Energy 112: 430 - 442.

Patteeuw, Dieter, Kenneth Bruninx, Alessia Arteconi, Erik Delarue, William D'haeseleer, and Lieve Helsen. 2015. "Integrated modeling of active demand response with electric heating systems coupled to thermal energy storage systems." Applied Energy 151: 306-319.

Patteeuw, Dieter, Gregor P Henze, and Lieve Helsen. 2016. "Comparison of load shifting incentives for low-energy buildings with heat pumps to attain grid flexibility benefits." Applied Energy 167: 80-92.

Santamouris, M., G. Mihalakakou, P. Patargias, N. Gaitani, K. Sfakianaki, M. Papaglastra, C. Pavlou, et al. 2007. "Using intelligent clustering techniques to classify the energy performance of school buildings." Energy and Buildings 39 (1): $45-51$.

UIUC, and LBNL. 2005. EnergyPlus engineering reference: the reference to EnergyPlus calculations. Technical report. US Department of Energy.

U.S. Energy Information Administration. 2009. "Residential Energy Consumption Survey (RECS)." Accessed: 2009, http: //www. eia.gov/consumption/residential/.

Ward Jr, Joe H. 1963. "Hierarchical grouping to optimize an objective function." Journal of the American statistical association 58 (301): 236244.

Yamaguchi, Y., Y. Shimoda, and M. Mizuno. 2007. "Proposal of a modeling approach considering urban form for evaluation of city level energy management." Energy and Buildings 39 (5): 580 592.

Yang, Junjing, Chao Ning, Chirag Deb, Fan Zhang, 
David Cheong, Siew Eang Lee, Chandra Sekhar, and Kwok Wai Tham. 2017. "k-Shape clustering algorithm for building energy usage patterns analysis and forecasting model accuracy improvement." Energy and Buildings 146: 27 37. 